\title{
Polysèmes
}

Revue d'études intertextuelles et intermédiales

\section{If Not, Not : citation et allégeance chez R.B. Kitaj et} T.S. Eliot

\section{Charlotte Gould}

\section{(2) OpenEdition}

Journals

Édition électronique

URL : http://journals.openedition.org/polysemes/3311

DOI : $10.4000 /$ polysemes.3311

ISSN : 2496-4212

Éditeur

SAIT

Référence électronique

Charlotte Gould, «If Not, Not : citation et allégeance chez R.B. Kitaj et T.S. Eliot », Polysèmes [En ligne], 19 | 2018, mis en ligne le 30 juin 2018, consulté le 20 avril 2019. URL : http://journals.openedition.org/ polysemes/3311; DOI : 10.4000/polysemes.3311

Ce document a été généré automatiquement le 20 avril 2019.

Polysèmes 


\title{
If Not, Not : citation et allégeance chez R.B. Kitaj et T.S. Eliot
}

\author{
Charlotte Gould
}

1 Lorsqu'en 1958 l'Américain R.B. Kitaj entame des études à la Ruskin School of Art d'Oxford, l'idée de s'installer en Angleterre s'inscrit pour lui dans la prestigieuse lignée des démarches d'auteurs et d'artistes américains ayant renoué avec le vieux continent, comme Henry James, Ezra Pound, T.S. Eliot ou James McNeill Whistler. Ce choix se révéla pourtant être le gage de son originalité lorsque, dans les années soixante, New York devint la Mecque de l'art contemporain. S'exiler, pour un artiste américain, signifiait renoncer à l'excitation qui régnait outre-Atlantique. À part, Kitaj le fut aussi par la forme en ceci qu'il éprouvait une certaine méfiance à l'égard de l'avant-garde. Associé à ses amis du Royal College of Art, David Hockney ou Allen Jones (au sujet desquels on employa le qualificatif pop, version britannique et peut-être plus distanciée qui précéda le Pop Art américain), Kitaj reste difficile à catégoriser. Sa principale particularité est qu'il a moins cherché son inspiration dans une réflexion sur l'histoire de l'art ou sur sa théorisation que dans la littérature. Il peint If Not, Not ${ }^{1}$ en 1976 alors que les artistes minimalistes s'interrogent sur les limites de leur art et font coïncider support et sujet. If Not, Not fait explicitement référence à The Waste Land (1922) de T.S. Eliot, Kitaj ayant, comme à son habitude, commenté lui-même son tableau et l'ayant inscrit dans une grille de références littéraires et philosophiques dans son catalogue d'exposition. Les deux œuvres se rejoignent en ce qu'elles sont des commentaires historiques sur les guerres $d u X X^{e}$ siècle, mais The Waste Land est loin d'être un prétexte ou une simple source de symboles; en effet, l'allégeance promise par Kitaj à Eliot va chercher à abolir la chronologie et à faire $\mathrm{du}$ poème et $\mathrm{du}$ tableau des œuvres sœurs, tendant vers l'interaction plus que la référence.

2 Avec If Not, Not, Kitaj tente comme Eliot d'appréhender un problème douloureux de l'histoire contemporaine. Quand The Waste Land fait état de la désolation du paysage européen (ses citations empruntent à différentes langues européennes) au sortir de la Première Guerre mondiale, If Not, Not recrée cet état de désolation après la découverte des atrocités commises pendant la Seconde Guerre mondiale. La représentation de la porte 
donnant sur le camp de la mort à Auschwitz, bâtisse impressionnante trônant sur la colline en arrière-plan, a pour Kitaj valeur de symbole, comme l'indique son caractère presque schématique, et ses lignes claires, sans nuances, qui semblent tracées à la règle ; le symbole de la capacité de l'homme à concevoir et à mettre en œuvre l'horreur absolue. Pourtant, ces événements faisant tous deux partie de l'histoire récente pour chacun des artistes, leur sens reste flou; leurs représentations s'attachent donc plus à recréer le sentiment qui les a accompagnés que les moments qui les ont marqués. Nous n'avons pas affaire à des narrations de ces deux guerres. Un élément comme l'eau sera utilisé de manière menaçante, voire morbide, dans "Death by Water ", la quatrième partie du poème, où Phlebas le Juif est entraîné par le courant. Dans "The Fire Sermon", les vers 187 à 190 semblent même prophétiques :

A rat crept softly through the vegetation

Dragging its slimy belly on the bank

While I was fishing in the dull canal

On a winter evening round behind the gashouse.

3 Chez Kitaj, l'eau sera stagnante, dans l'ombre. Le thème principal du tableau trouve encore sa source littérale dans The Waste Land: «White bodies naked on the low damp ground » (vers 193). Cette eau au caractère ambivalent, entre vie (le narrateur du poème $y$ pêche) et mort (elle rejette les cadavres des victimes de la guerre) n'est pas le seul symbole à être détourné. Pour trouver du sens, chaque artiste a bousculé l'imagerie traditionnelle. Chez Eliot « April is the cruellest month » (vers 1), le printemps n'annonce pas le retour à la vie mais au contraire le choc provoqué par la remontée des mauvais souvenirs qui avaient été enfouis :

Winter kept us warm, covering

Earth in forgetful snow... (vers 5-6)

4 Au vers 8 (« Summer surprised us »), les sentiments habituellement associés aux saisons sont également intervertis. L'ordre naturel des choses a été perverti par l'homme depuis qu'il a permis la destruction de la jeune génération à la guerre. Chez Kitaj, le tableau représentant un paysage est détourné de son caractère habituellement paisible. L'influence est celle de Giorgione et de sa Tempesta - le bébé au sein de Giorgione est, chez Kitaj, auprès de son père. Le lieu est alors celui d'un croisement : un paysage européen pour le déroulement d'une histoire juive. Au centre du tableau, à la fois l'eau qui rappelle Giorgione et le bélier qui raconte l'akedah, l'histoire d'Isaac attaché à l'autel du sacrifice et qui sera finalement remplacé par l'animal. Paradigme du martyre juif, l'histoire est aussi une mine d'interprétations kabbalistiques (Kierkegaard l'a démontré dans Crainte et tremblements). Les arbres exotiques et le coucher de soleil côtoient les chambres à gaz. Kitaj avait été marqué par les récits de gens ayant refait le voyage effectué par les déportés pour arriver aux camps ; tous insistaient sur la beauté du paysage. Buchenwald fut érigé sur cette même colline où Goethe avait coutume de se promener en compagnie d'Eckermann. Le choc de la découverte de l'horreur est donc accentué par le paysage idyllique qui se trouve alentour. Tout comme Eliot construit son poème avec les fragments des plus beaux textes qu'il connait, tel le « Good night ladies, good night, sweet ladies, good night, good night » (vers 172) d'Ophélie, Kitaj décrit la désolation en faisant de l'antichambre de l'enfer un endroit plutôt paradisiaque, ou du moins une de ces visions un peu convenues du paradis où un soleil couchant est beau par définition.

5 Kitaj et Eliot veulent créer du sens artistique à partir du chaos de la guerre. L'histoire moderne est narrée à travers de nouveaux supports et le récit de la guerre s'adapte pour prendre de nouvelles formes. Les livres, les photos sont rejoints par le cinéma et les plans 
cinématographiques s'ajoutent aux autres sources et aux autres formes de représentation utilisées par Eliot pour faire de The Waste Land une sorte de montage de différents matériaux, un nouveau poème pour une nouvelle guerre dans un nouveau monde. Cette modernité prend chez Kitaj la forme de la figure humaine, ce parti pris humain allant à l'encontre des courants artistiques de l'époque, tel le Conceptualisme. La figuration défendue par Kitaj, dans sa propre peinture ou lorsqu'il organise une exposition de groupe à la Hayward Gallery intitulée "The Human Clay " en 1976, est en effet un leitmotiv de la création contemporaine et il va jusqu'à créer des personnages, au sens littéraire plutôt que pictural, qui interviennent de façon récurrente dans ses tableaux (comme Shakespeare fait revenir Falstaff) ${ }^{2}$. Le plus remarquable est Joe Singer que l'on croise dans The Listener (1980) ou dans The Jewish Rider (1984), le nom étant celui d'un petit ami de jeunesse de sa mère. Dans If Not, Not, le personnage connu, à gauche au premier plan d'un tableau où les corps sont omniprésents, est l'homme à lunettes habillé de gris et porteur d'une prothèse auditive. L'interprétation selon laquelle ce personnage représenterait Eliot semble pouvoir être contredite par le fait qu'on le retrouve dans des tableaux ne se référant pas explicitement au poète, et qu'on avait à l'époque conseillé à Kitaj de porter un tel appareil ; il se mettrait donc en scène, comme le costume de ville presque anachronique le laisse suggérer. La prothèse auditive fait de lui une sorte d'espion, du moins un témoin qui tente de mieux entendre ce qui se passe autour de lui et de mieux voir grâce aux lunettes qu'il porte. Le peintre lui-même est une sorte d'espion face à cette réalité de la guerre dont il veut appréhender le sens. La vision est donc ici, comme l'ouie, synonyme de compréhension; dire « je vois » implique plus que la simple capacité à voir, et ce thème central de la vision trouve sa source chez Eliot. The Waste Land est tout autant un paysage intérieur et métaphorique que la construction d'une terre morte. Vue sensorielle et vue spirituelle se mélangent. Dans « The Fire Sermon », Tirésias est aveugle mais ses visions prophétiques sont avérées, elles sont plus claires que si elles avaient été réellement vues. Madame Sosostris, elle, n'est qu'une fausse voyante, les mystères ne lui sont pas révélés :

...I do not find

The Hanged man...(vers 54-55)

6 La foule refuse de voir : « And each man fixed his eyes before his feet » (vers 65), et toutes les femmes se regardent soit avec vanité, soit avec indifférence dans le miroir sans jamais trouver le reflet juste : "Her brain allows one half-formed thought to pass " (vers 251) jusqu'à ce que dans la dernière partie, "What the Thunder Said", la vue ne soit plus distincte de l'hallucination:

Who is the third who walks always beside you

When I count, there are only you and I together. (vers 359-360)

7 De même l'esprit se dissout totalement: "Shall I at least set my lands in order? » (vers 425). Ces quêtes de vision claire, de compréhension, rencontrent ainsi des obstacles, suggérant une idée de soumission passive des différents personnages du poème et du tableau plutôt que la vitalité, ce qui ajoute à la désolation du paysage.

Si Kitaj choisit le truchement de The Waste Land pour traiter des conséquences de la guerre plutôt que de s'inspirer directement des faits, c'est que la poésie et la littérature représentent pour lui une toile de fond essentielle. À gauche du tableau, au pied de la colline, des livres sont éparpillés qui le rappellent. Cette position n'est d'ailleurs pas seulement présentée comme un clin d'œil mais affirmée tel un manifeste dans le catalogue de son exposition de 1963 en citant Horace et son fameux : « As in painting, so it 
is in poetry " (Kitaj 1963, 2), puis en 1965 dans une interview à Time Magazine : «For me, books are what trees are for the landscape painter » (Kitaj 1965, 42), où l'on trouve déjà l'analogie entre arbre et livre de If Not, Not. On se trouve donc au-delà de l'art évoquant sa propre nature. Pourtant le problème se pose alors pour Kitaj qu'il doit prendre garde à ne pas devenir dépendant de l'aura de ses sources pour donner de la profondeur à ses tableaux, surtout lorsque ses références sont aussi prestigieuses que T.S. Eliot. Comment parvenir à se servir de références littéraires sans en devenir l'esclave?

9 La critique souvent formulée à l'encontre de R.B. Kitaj est qu'il est un artiste trop littéraire, qu'il cite plus qu'il n'invente lui-même. Sa relation aux références littéraires est toutefois plus complexe, comme l'indique le titre If Not, Not. Celui-ci est exemplaire de la conscience politique et sociale qui traverse l'œuvre de Kitaj car il est extrait de l'ancienne formule des vœux prononcés au moment du couronnement d'un Aragon :

We who are as good as you swear to you who are no better than we, to accept you as our king, provided you observe all our liberties and laws; but if not, not. ${ }^{3}$

10 Cette définition de la relation entre souverain et sujets (nobles, bien sûr) dans les royaumes d'Espagne médiévale éclaire la conception de Kitaj sur l'allégeance à porter à son maître. Ainsi, lorsqu'il reconnaît Eliot comme référence, il n'opère pas une simple transposition du texte à l'image ; l'illustration est impossible car l'allégeance promise au poète réfute la soumission. Le fait qu'il se considère lui-même comme un héritier du Surréalisme explique qu'il travaille plus par associations que par références, voire par hommages qui seraient mortifères. Le titre de l'un de ses tableaux s'inspire du premier Manifeste du Surréalisme de Breton de 1924 : Certain Forms of Association Neglected Before, et c'est ce travail par association qui annule la précédence de The Waste Land sur If Not, Not. Eliot lui-même, dans son travail de croisement de citations, s'apparente à la Sibylle, cette prophétesse de légende qui répondait aux questions de ses visiteurs en jetant à terre une poignée de feuilles portant chacune une lettre et que la personne en quête de sagesse devait ensuite arranger elle-même dans un ordre cohérent. Par une mise en abyme, les personnages du poème vont consulter Madame Sosostris.

11 Afin de mettre en œuvre cette allégeance, Kitaj dépasse la référence pour chercher l'identité des formes. The Waste Land fonctionne comme une structure d'allusions, de citations, mélangeant les registres familiers et lyriques (l'histoire de la fille à la jacinthe contraste avec celle de Lil : "Now Albert's coming back, make yourself a bit smart", vers 142) et de continuum entre le passé et le présent. Eliot utilise les techniques de l'ironie et du paradoxe pour éviter l'aliénation d'une unité de sentiment et pour ne pas réduire l'œuvre à une formule. Visuellement, cela donne, dans If Not, Not une juxtaposition d'éléments disparates, de choses hors de contexte, tels ces palmiers exotiques à Auschwitz. Les onomatopées d'Eliot ("Jug Jug », vers 103 ou " Co co rico co co rico ", vers 392) correspondent à des moments d'abstraction chez Kitaj, par exemple dans la forme de la colline ou des arbres. Les fragments d'œuvres connues se retrouvent dans le tableau sous la forme du buste de Matisse brisé. Le ton est dans les deux cas ambivalent: chez Eliot, une vision angoissée est rendue à travers des vers courts et légers; chez Kitaj, l'horreur prend lieu dans le cadre de la tradition du paysage, accompagnée de couleurs chaudes. Les rejets du poème ont pour écho la technique du collage. If Not, Not rappelle un arrêt sur image de cinéma qui supposerait une continuité narrative, un contexte, et on trouve chez Eliot ce sentiment d'ordre simultané, comme si l'histoire narrée n'avait pas de fil mais s'appréhendait globalement. Il s'agit de la conception de la poésie exposée par Coleridge : 
The common end of all narrative, nay, of all Poems, is to convert a series into a whole: to make those events, which in real or imagined History move on in a strait line, assume to our understandings a circular motion-the snake with its tail in its mouth. (Griggs 218)

Il n'y a pas, ni dans le poème, ni dans le tableau, de liens logiques ; la narration repose sur des suggestions élaborées, des échos. Dans The Waste Land, ce système de rappels abolit les contrastes sociaux entre la femme de "A Game of Chess » et Lil dans son pub cockney, entre la reine et le pion; toutes deux habitent un désert érotique où les parfums sont synthétiques et où les fonctions naturelles sont handicapées, soit par l'hystérie, soit par les médicaments («It's them pills I took», vers 159), et au sujet de toutes deux Eliot emploie le mot "antique " "You ought to be ashamed, I said, to look so antique " vers 156 et « Above the antique mantel was displayed... » vers 97, dans le boudoir de la grande dame).

13 En dépassant la référence et en s'inspirant des formes utilisées par Eliot, Kitaj veut comme le grand poète mettre en images les sources de la conscience moderne, comme lui il veut briser la tradition afin de la restructurer et de la revitaliser. On trouve chez les deux une grande quantité d'allusions littéraires explicites ou non, pourtant aucun ne cherche à s'éloigner de la vie car comme le dit Eliot lui-même : "The ordinary life of ordinary cultivated people is a mush of literature and life $»^{4}$. L'art aiguise et étend notre conscience du sens de l'expérience. Ainsi le poème comme le tableau s'inspirent-ils avant tout de la vie et Kitaj le souligne pour faire disparaittre l'antécédence de The Waste Land. L'œuvre plus récente peut transformer le contenu de sa référence en changeant la perception qu'en a le public, car ce dernier va ici inclure la vision de Kitaj dans sa relecture de The Waste Land. Les œuvres ne se suffisant pas à elles-mêmes, l'artiste crée ses précurseurs; et là, Kitaj remonte à un ami d'Eliot qui devient une inspiration pour tous les deux : il reprend et s'approprie la différence établie par Pound entre le symbole qui épuise ses références et un signe ou une marque de quelque chose qui sans cesse renouvelle la référence. Les poèmes seraient ainsi des signes, le plus souvent des signes incompris (Livingstone 17). Pour renouveler, il faut donc se détacher du symbolisme, et ce n'est pas symboliquement que Kitaj reprend Eliot, mais dans la signifiance de ses propres reprises, ce qui est très clair en ce qui concerne l'imagerie empruntée à Joseph Conrad et à Heart of Darkness où la rivière représente la pénétration de la corruption. Conrad intervient pour tous les deux comme une référence littéraire qui informe la vie et fait partie de l'expérience plus que d'une vision figée de la littérature.

14 La critique qui émerge face à cette interaction entre deux œuvres a trait au problème des notes. En effet, Kitaj fut un peintre original en ce qu'il a exposé ses tableaux accompagnés de longues notices explicatives, censées être non pas des exégèses, mais bien un texte littéraire permettant une véritable continuité formelle entre texte et image, une unité qui en fait une seule et même œuvre : «I wish to write a preface, or [...] better term, epilogue, for many of my pictures $»^{5}$. L'abondance de notices accompagnant les œuvres lors de sa grande rétrospective à la Tate Gallery de Londres en 1994, leur caractère direct, personnel, déclenchèrent une salve de critiques, parfois tout aussi personnelles. Ce rejet violent d'œuvres principalement récentes acheva de consommer son divorce d'avec la critique. De son côté, Eliot avait fait suivre The Waste Land de notes presque exhaustives sur les allusions du texte à des œuvres célèbres, mais les avait ensuite amèrement regrettées, comme si elles avaient été une excroissance de son poème, une imperfection. Il fallait pourtant lever le voile de mystère de ces références pour écarter un danger qui menaçait le lecteur, celui qui consiste à penser qu'on a découvert du sens alors que l'on 
vient seulement d'identifier une allusion. Mais on pourrait alors reprocher à Kitaj d'avoir opéré une simple équivalence picturale à ce travail de notes pour créer une œuvre de la référence, un tableau qui ne serait élaboré que par collage, assemblage de morceaux de choix. Pourtant Kitaj ne s'inspire en fait pas plus des notes d'Eliot que véritablement de son poème, parce qu'il y a chez Eliot de vrais et de faux parallèles avec ses sources, que le thème d'ordre et d'unité de son poème est la disharmonie qui y règne et que Kitaj est parvenu à restituer. Kitaj s'attache à évoquer la présence des gens déracinés, des perdants dans la lutte pour le pouvoir, du Juif Errant; la diaspora est pour lui l'emblème du caractère diffus de la culture au $\mathrm{XX}^{\mathrm{e}}$ siècle, elle symbolise cette disharmonie que présentait Eliot.

15 Kitaj opère en fait une déconstruction littéraire en refusant à un texte la possibilité d'avoir une seule signification; il rapproche en cela son art de la tradition juive de la midrash, manière d'interpréter les textes sacrés qui consiste à accumuler des notes de critique sur chaque ligne du texte même, créant une sorte de palimpseste sans que l'on parvienne à un accord quant à un sens définitif. Cette technique évite à Kitaj de devenir illustrateur de la littérature, sans pour autant qu'il tombe dans l'écueil de la congestion, grâce à la gamme picturale qu'il parcourt et qui va des détails ironiques aux élans lyriques (pour emprunter un terme littéraire). Ainsi, lorsqu'est dépassé le simple rapport de référence entre les deux œuvres et qu'on est parvenu à abolir toute subordination, on peut considérer que If Not, Not est la forme prise par la méditation de Kitaj sur The Waste Land et sur son rythme.

Avec If Not, Not et The Waste Land on a donc affaire à deux palimpsestes en réponse aux énigmes posées par la culture moderne. Kitaj comme Eliot peuvent ainsi commenter la culture de reproduction sans y succomber, sans transférer leurs motifs à partir d'une culture de masse, car ils créent de la nouveauté, en surimpression. La guerre mondiale se reproduit après The Waste Land et Kitaj peut réutiliser pour réinventer à la fois la compréhension du présent et celle de ce poème du passé. Eliot avait d'abord pensé mettre un passage de Heart of Darkness en épigraphe au poème : "The horror! The horror! ». Il s'agit de la réaction de Kurtz au moment où il a l'impression d'avoir atteint la connaissance totale, et si, ni pour Eliot, ni pour Kitaj, on ne parvient à appréhender le sens total de choses, l'impression de chaos est un début de réponse subjective car tout s'ajoute en surimpression, transformant la compréhension de ce qui existait déjà.

17 L'absence de frontières entre production écrite et visuelle qui avait fait enrager une critique londonienne dépossédée de ses prérogatives était un hommage de Kitaj à son pays d'accueil et à l'influence d'endroits comme le Warburg Institute et son travail d'iconographie. Blessé par les attaques et convaincu que le décès prématuré de sa seconde femme, la peintre Sandra Fisher, intervenu quinze jours après la fermeture de l'exposition, était dû à leur férocité, Kitaj quitte le continent de la mémoire pour rejoindre celui de sa naissance, de la nouveauté et de l'oubli ${ }^{6}$. Installé à Los Angeles, le premier Académicien royal américain depuis John Singer Sargent se suicide en 2007. La critique britannique reste sans voix, encore gênée par ses excès. C'est dans le hall d'entrée principal de la British Library que l'architecte Colin St John Wilson a décidé d'installer une tapisserie reprenant If Not, Not. La bibliothèque londonienne est ainsi devenue le lieu magistral où If Not, Not a pris la forme d'un monument à l'artiste, monument qui reconnait la fraternité de son œuvre picturale avec les livres qui partagent ce même lieu public. 


\section{BIBLIOGRAPHIE}

Corbett, David Peters. « Authority and Visual Experience: Word and Image in R.B. Kitaj ». Critical Kitaj. James Aulich et John Lynch (eds.). Manchester: Manchester UP, 2000.

Eliot, T.S. The Waste Land (1922). Collected Poems 1909-1963. London: Faber and Faber, 1963.

Griggs, E.L. Collected Letters of S. T. Coleridge. Vol. II, 1959.

Hughes, Robert. Barcelona, London: The Harvill Press, 2001.

Kitaj, Ronald Brooks. Pictures with Commentary, Pictures without Commentary. London: Marlborough Gallery, 1963.

Kitaj, Ronald Brooks. « Painting: Literary Collage ». New York : Time, 19 février 1965.

Livingstone, Marco. R.B. Kitaj. Oxford: Phaidon, 1985.

\section{NOTES}

1. Ronald Brooks Kitaj, If Not, Not, 1975-1976, $152.4 \mathrm{~cm}$ x $152.4 \mathrm{~cm}$, oil and black chalk on canvas, Scottish National Gallery of Modern Art, Edinburgh.

2. C'est dans l'introduction du catalogue de l'exposition « The Human Clay » que Kitaj évoque et baptise par la même occasion « the School of London ».

3. Serment d'allégeance catalan et aragonais, interdit par les Bourbons en 1714 : "Nós, que valem tant com vós per separat, i junts més que vós, us investim sobirà i us jurem lleialtat per tal que ens protegiu, defenseu i treballeu pel nostre progrés, i si no, no." (Hughes 105).

4. T.S. Eliot, préface à Selected Poems of Ezra Pound (London, 1928).

5. Kitaj cité par David Peters Corbett (dans Aulich 44).

6. Le tableau The Killer-Critic Assassinated by His Widower, Even de 1997 formule ses griefs contre ceux qu'il considère comme les meurtriers de Sandra et contre leurs impératifs postduchampiens. 